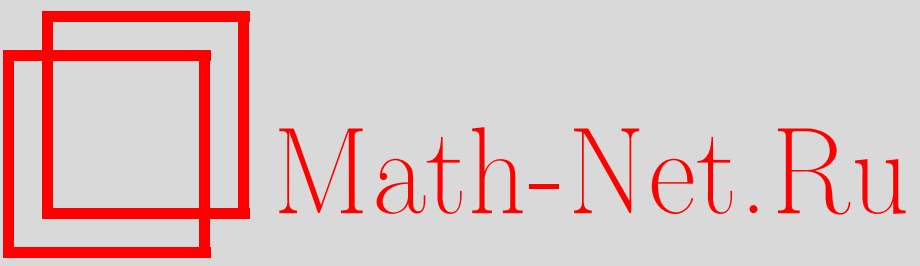

Х. А. Назаров, У. А. Розиков, О периодических гиббсовских мерах модели Изинга с конкурирующими взаимодействиями, ТMФ, 2003, том 135, номер 3, 515-523

DOI: https://doi.org/10.4213/tmf201

Использование Общероссийского математического портала Math-Net.Ru подразумевает, что вы прочитали и согласны с пользовательским соглашением

http://www.mathnet.ru/rus/agreement

Параметры загрузки:

IP : 54.164 .48 .24

26 апреля 2023 г., 15:59:20 
ТЕОРЕТИЧЕСКАЯ

И МАТЕМАТИЧЕСКАЯ

ФИЗИКА

Том 135, № 3

июнь, 2003

(C) 2003 г.

\author{
Х. А. Назаров ${ }^{*}$, У.А. Розиков ${ }^{\dagger}$
}

\title{
О ПЕРИОДИЧЕСКИХ ГИББСОВСКИХ МЕРАХ МОДЕЛИ ИЗИНГА С КОНКУРИРУЮЩИМИ ВЗАИМОДЕЙСТВИЯМИ
}

Для модели Изинга с конкурируюшими взаимодействиями на дереве Кэли второго порядка получен оператор, соответствующий периодическим распределениям Гиббса с периодом два. Найдены инвариантные подмножества этого оператора, с применением которых описаны периодические гиббсовские распределения.

Ключевые слова: дерево Кэли, конфигурация, предельная мера Гиббса, периодическая мера Гиббса.

\section{1. ВВЕДЕНИЕ}

Работа посвяшена изучению модели Изинга с конкурируюшими взаимодействиями на первых и вторых соседях на дереве Кэли. Дерево Кэли $\Gamma^{k}=(V, L), k \geqslant 1$, есть бесконечное дерево, т.е. граф̆ без циклов, из каждой вершины которого выходят ровно $k+1$ ребер ( $V$ есть множество вершин $\Gamma^{k}, L$ - множество его ребер).

В работе [1] доказано, что $\Gamma^{k}$ можно представить как $G_{k}-$ свободное произведение $k+1$ циклических групп второго порядка. Если гиббсовское распределение инвариантно относительно некоторой подгруппы конечного индекса $G_{k}^{*}$ группы $G_{k}$, то оно называется $G_{k}^{*}$-периодическим. Достаточно широкий класс нормальных делителей конечного индекса группы $G_{k}$ описан в работе [2]. В работе [3] изучена структура разбиений дерева Кэли; доказано также, что существует три $H_{0}$-периодических распределения Гиббса для неоднородной модели Изинга, где $H_{0}$ - нормальный делитель конечного индекса. В работе [4] построено несчетное число непериодических распределений Гиббса с помошью распределений, полученных в работе [3]. Но если в модели происходят конкурируюшие взаимодействия, задача описания периодических распределений Гиббса нелегка. Дело в том, что в этой ситуации возникает нелинейный оператор $W$, отображающий $\mathbb{R}^{r}$ в себя ( $r$ - индекс нормального делителя), и задача состоит в описании

\footnotetext{
${ }^{*}$ Самаркандский государственный архитектурный институт, Самарканд, Республика Узбекистан

${ }^{\dagger}$ Институт математики АН РУз, Ташкент, Республика Узбекистан
} 
неподвижных точек этого оператора. Если известны инвариантные подмножества оператора $W$, то, рассматривая сужения этого оператора на инвариантные подмножества, можно надеяться на получение некоторых неподвижных точек, так как вид оператора в этом случае упрошается.

Если $l=\langle x, y\rangle \in L$, то $x, y \in V$ называются соседними. Вершины $x, z \in V$ называются вторыми соседями и обозначаются $\rangle x, z\langle$, если сушествует $y \in V$ такое, что $\langle x, y\rangle$ и $\langle y, z\rangle$.

Пусть $\Phi=\{-1,1\}$ и $\sigma \in \Phi^{V}$, т.е. $\sigma=\left\{\sigma_{x} \in \Phi: x \in V\right\}$. Рассмотрим гамильтониан

$$
H(\sigma)=-J_{1} \sum_{\langle x, y\rangle} \sigma_{x} \sigma_{y}-J_{2} \sum_{\rangle x, y\langle} \sigma_{x} \sigma_{y}
$$

где $J_{1}, J_{2} \in \mathbb{R}, \sigma_{x}= \pm 1$ для любого $x \in V$.

Модель (1) назьвается моделью Изинга с конкурируюшими взаимодействиями.

Обычным образом вводятся понятия предельного распределения Гиббса модели (1), чистой фазы и т.д.

В статье [5] решена задача описания трансляционно-инвариантных гиббсовских мер модели (1). В работе [6] модель (1) исследована на физическом уровне и при конкретных значениях параметров $J_{1}, J_{2}$ дана фазовая диаграмма состояния системы.

В настоящей работе мы ограничимся случаем $k=2$. Будем изучать оператор, соответствующий модели (1) в случае нормального делителя $G_{k}^{(2)}$ индекса 2, который является подгруппой, состоящей из слов четной длины.

Цель работы - найти инвариантные подмножества рассматриваемого оператора и, применяя их, описать периодические гиббсовские меры.

\section{2. ОПЕРАТОР, СООТВЕТСТВУЮЩИЙ $G_{k}^{(2)}$-ПЕРИОДИЧЕСКИМ МЕРАМ}

Пусть $V$ есть множество вершин $\Gamma^{2}$. Пронумеруем точки множества $V=$ $\{0, i, i j, i j k, \ldots ; i=1,2,3, j, k, l, \cdots=1,2\}$ следующим образом. Выберем произвольную точку 0, ближайшим соседним вершинам этой точки поставим в соответствие числа $1,2,3$; ближайшим соседним вершинам вершин $i, i=1,2,3$, отличным от 0 , поставим в соответствие пары чисел $i j$, где $j=1,2$; ближайшим соседним вершинам вершин $i j$, не имеющим номеров, поставим в соответствие тройку $i j k$, где $k=1,2$, и т.д.

Всякую функцию $\sigma=\left\{\sigma_{x}, x \in V\right\}$, определенную на множестве $V$ и принимаюшую значения $\sigma_{x}= \pm 1$, будем называть конфигурацией в $V$, а совокупность всех таких конфигураций обозначим через $\Omega$.

Расстояние $d(x, y), x, y \in V$, есть число ребер пути из $x$ в $y$.

Обозначим $W_{n}=\{x \in V: d(0, x)=n\}, V_{n}=\{x \in V: d(0, x) \leqslant n\}, K=J_{1} / T, K^{\prime}=$ 
$J_{2} / T$

$$
\begin{gathered}
Y^{i}\left(\sigma_{0}, \sigma_{i}\right)=\sum_{\sigma_{i j}} \exp \left(K^{\prime} \sigma_{i 1} \sigma_{i 2}\right) \prod_{j} \exp \left(K \sigma_{i} \sigma_{i j}+K^{\prime} \sigma_{0} \sigma_{i j}\right) Y^{i j}\left(\sigma_{i}, \sigma_{i j}\right), \\
Y^{i j}\left(\sigma_{i}, \sigma_{i j}\right)=\sum_{\sigma_{i j k}} \exp \left(K^{\prime} \sigma_{i j 1} \sigma_{i j 2}\right) \prod_{k} \exp \left(K \sigma_{i j} \sigma_{i j k}+K^{\prime} \sigma_{i} \sigma_{i j k}\right) Y^{i j k}\left(\sigma_{i j}, \sigma_{i j k}\right), \\
\frac{Y^{i_{1} i_{2} \ldots i_{n}}(\sigma,-1)}{Y^{i_{1} i_{2} \ldots i_{n}}(\sigma, 1)}=x^{\sigma}\left(i_{1} i_{2} \ldots i_{n}\right), \\
\theta_{1}=e^{-2 K}, \quad \theta_{2}=e^{-2 K^{\prime}} .
\end{gathered}
$$

Пусть $\sigma_{n}=\left\{\sigma_{x}, x \in V_{n}\right\}$ - конфигурация на $V_{n}$. Совокупность всех таких конфигураций обозначим через $\Omega_{n}$.

Введем в пространстве $\Omega_{n}$ меру $\mu_{n}$ следуюшим образом:

$$
\mu_{n}\left(\sigma_{n}\right)=Z_{n}^{-1} e^{-\beta H_{n}\left(\sigma_{n}\right)} \prod_{i_{1} i_{2} \ldots i_{n} \in W_{n}} Y^{i_{1} i_{2} \ldots i_{n}}\left(\sigma_{i_{1} i_{2} \ldots i_{n-1}}, \sigma_{i_{1} i_{2} \ldots i_{n}}\right),
$$

где $\beta=1 / T$ ( $T>0$ - температура), $Z_{n}^{-1}$ - нормируюший множитель и

$$
H_{n}\left(\sigma_{n}\right)=-J_{1} \sum_{\langle x, y\rangle: x, y \in V_{n}} \sigma_{x} \sigma_{y}-J_{2} \sum_{\left\langle x, y\left\langle: x, y \in V_{n}\right.\right.} \sigma_{x} \sigma_{y} .
$$

Условия согласованности для $\mu_{n}$ определяются равенством

$$
\sum_{\sigma^{(n)}} \mu_{n}\left(\sigma_{n-1}, \sigma^{(n)}\right)=\mu_{n-1}\left(\sigma_{n-1}\right)
$$

где $\sigma^{(n)}=\left\{\sigma_{x}, x \in W_{n}\right\}$.

Теорема 1. Мерь (2) удовлетворяют условиям (3) тогда и только тогда, когда для функиии $x^{\sigma}(t), \quad t \in V$, выполняется равенство

$$
x^{\sigma}(t)=G^{\sigma}\left(x^{+}(t 1), x^{+}(t 2)\right) \Phi^{\sigma}\left(x^{-}(t 1), x^{-}(t 2)\right) \prod_{j=1}^{2} F\left(x^{-}(t j 1), x^{-}(t j 2)\right),
$$

əде $\sigma= \pm 1$,

$$
\begin{aligned}
F(x, y) & =\frac{\theta_{1}^{2} \theta_{2}^{2}+\theta_{1} \theta_{2}^{2}(x+y)+x y}{\theta_{1}^{2}+\theta_{1} \theta_{2}^{2}(x+y)+\theta_{2}^{2} x y}, \\
G^{\sigma}(x, y) & =\frac{x y}{1+\theta_{1} \theta_{2}^{\sigma+1}(x+y)+\theta_{1}^{2} \theta_{2}^{2 \sigma} x y}, \\
\Phi^{\sigma}(x, y) & =\frac{\theta_{1}^{2}+\theta_{1} \theta_{2}^{\sigma+1}(x+y)+\theta_{2}^{2 \sigma} x y}{x y} .
\end{aligned}
$$


ДоКАЗАТЕЛЬСтво. Эта теорема есть следствие теоремы 2.2 из работы [5].

Таким образом, каждой предельной гиббсовской мере $\mu$ на $\Omega=\Phi^{V}$ сопоставляется совокупность векторов

$$
h=\left\{x(t)=\left(x^{-}(t), x^{+}(t)\right): t \in G_{2}\right\}
$$

координаты которых удовлетворяют равенству (4). Но описание решений функционального уравнения (4) - весьма сложная задача. Поэтому естественно сначала искать те из них, которые принимают лиш конечное число значений.

Для описания $G_{2}^{(2)}$-периодических мер предположим, что

$$
x^{\sigma}(t)= \begin{cases}x_{1}^{\sigma}, & \text { если } t \in G_{2}^{(2)} \\ x_{2}^{\sigma}, & \text { если } t \in G_{2} \backslash G_{2}^{(2)} .\end{cases}
$$

Тогда из (4) получим

$$
\left\{\begin{array}{l}
x_{1}^{+}=\frac{x_{2}^{+}}{x_{2}^{-}} \frac{\theta_{1}^{2}+2 \theta_{1} \theta_{2}^{2} x_{2}^{-}+\theta_{2}^{2}\left(x_{2}^{-}\right)^{2}}{1+2 \theta_{1} \theta_{2}^{2} x_{2}^{+}+\theta_{1}^{2} \theta_{2}^{2}\left(x_{2}^{+}\right)^{2}} F_{1}\left(x_{1}^{-}, x_{1}^{+}\right), \\
x_{1}^{-}=\frac{x_{2}^{+}}{x_{2}^{-}} \frac{\theta_{1}^{2} \theta_{2}^{2}+2 \theta_{1} \theta_{2}^{2} x_{2}^{-}+\left(x_{2}^{-}\right)^{2}}{\theta_{2}^{2}+2 \theta_{1} \theta_{2}^{2} x_{2}^{+}+\theta_{1}^{2}\left(x_{2}^{+}\right)^{2}} F_{1}\left(x_{1}^{-}, x_{1}^{+}\right), \\
x_{2}^{+}=\frac{x_{1}^{+}}{x_{1}^{-}} \frac{\theta_{1}^{2}+2 \theta_{1} \theta_{2}^{2} x_{1}^{-}+\theta_{2}^{2}\left(x_{1}^{-}\right)^{2}}{1+2 \theta_{1} \theta_{2}^{2} x_{1}^{+}+\theta_{1}^{2} \theta_{2}^{2}\left(x_{1}^{+}\right)^{2}} F_{1}\left(x_{2}^{-}, x_{2}^{+}\right), \\
x_{2}^{-}=\frac{x_{1}^{+}}{x_{1}^{-}} \frac{\theta_{1}^{2} \theta_{2}^{2}+2 \theta_{1} \theta_{2}^{2} x_{1}^{-}+\left(x_{1}^{-}\right)^{2}}{\theta_{2}^{2}+2 \theta_{1} \theta_{2}^{2} x_{1}^{+}+\theta_{1}^{2}\left(x_{1}^{+}\right)^{2}} F_{1}\left(x_{2}^{-}, x_{2}^{+}\right),
\end{array}\right.
$$

где

$$
F_{1}(x, y)=F_{1}\left(x, y, \theta_{1}, \theta_{2}\right)=\frac{\theta_{2}^{2}+2 \theta_{1} \theta_{2}^{2} y+\theta_{1}^{2} y^{2}}{1+2 \theta_{1} \theta_{2}^{2} y+\theta_{1}^{2} \theta_{2}^{2} y^{2}} \cdot \frac{\theta_{1}^{2} \theta_{2}^{2}+2 \theta_{1} \theta_{2}^{2} x+x^{2}}{\theta_{1}^{2}+2 \theta_{1} \theta_{2}^{2} x+\theta_{2}^{2} x^{2}}
$$

Рассмотрим оператор $W: \mathbb{R}^{4} \rightarrow \mathbb{R}^{4}$ :

$$
\left\{\begin{array}{l}
y_{1}^{+}=\frac{x_{2}^{+}}{x_{2}^{-}} \frac{\theta_{1}^{2}+2 \theta_{1} \theta_{2}^{2} x_{2}^{-}+\theta_{2}^{2}\left(x_{2}^{-}\right)^{2}}{1+2 \theta_{1} \theta_{2}^{2} x_{2}^{+}+\theta_{1}^{2} \theta_{2}^{2}\left(x_{2}^{+}\right)^{2}} F_{1}\left(x_{1}^{-}, x_{1}^{+}\right), \\
y_{1}^{-}=\frac{x_{2}^{+}}{x_{2}^{-}} \frac{\theta_{1}^{2} \theta_{2}^{2}+2 \theta_{1} \theta_{2}^{2} x_{2}^{-}+\left(x_{2}^{-}\right)^{2}}{\theta_{2}^{2}+2 \theta_{1} \theta_{2}^{2} x_{2}^{+}+\theta_{1}^{2}\left(x_{2}^{+}\right)^{2}} F_{1}\left(x_{1}^{-}, x_{1}^{+}\right), \\
y_{2}^{+}=\frac{x_{1}^{+}}{x_{1}^{-}} \frac{\theta_{1}^{2}+2 \theta_{1} \theta_{2}^{2} x_{1}^{-}+\theta_{2}^{2}\left(x_{1}^{-}\right)^{2}}{1+2 \theta_{1} \theta_{2}^{2} x_{1}^{+}+\theta_{1}^{2} \theta_{2}^{2}\left(x_{1}^{+}\right)^{2}} F_{1}\left(x_{2}^{-}, x_{2}^{+}\right), \\
y_{2}^{-}=\frac{x_{1}^{+}}{x_{1}^{-}} \frac{\theta_{1}^{2} \theta_{2}^{2}+2 \theta_{1} \theta_{2}^{2} x_{1}^{-}+\left(x_{1}^{-}\right)^{2}}{\theta_{2}^{2}+2 \theta_{1} \theta_{2}^{2} x_{1}^{+}+\theta_{1}^{2}\left(x_{1}^{+}\right)^{2}} F_{1}\left(x_{2}^{-}, x_{2}^{+}\right) .
\end{array}\right.
$$

Заметим, что система (5) - это уравнение $W(x)=x, x \in \mathbb{R}^{4}$. Решения этой системы определяют периодические распределения Гиббса. 


\section{3. ОБ ИНВАРИАНТНЫХ МНОЖЕСТВАХ ОТОБРАЖЕНИЯ $W$}

Обозначим

$$
\begin{aligned}
& A=\left\{\left(x_{1}^{-}, x_{1}^{+}, x_{2}^{-}, x_{2}^{+}\right) \in \mathbb{R}^{4}: x_{1}^{-}=x_{2}^{-}, x_{1}^{+}=x_{2}^{+}\right\}, \\
& B=\left\{\left(x_{1}^{-}, x_{1}^{+}, x_{2}^{-}, x_{2}^{+}\right) \in \mathbb{R}^{4}: x_{1}^{-}=\frac{1}{x_{1}^{+}}, x_{2}^{-}=\frac{1}{x_{2}^{+}}\right\}, \\
& C=\left\{\left(x_{1}^{-}, x_{1}^{+}, x_{2}^{-}, x_{2}^{+}\right) \in \mathbb{R}^{4}: x_{1}^{-}=\frac{1}{x_{2}^{+}}, x_{1}^{+}=\frac{1}{x_{2}^{-}}\right\} .
\end{aligned}
$$

Tеорема 2. Множества $A, B, C$ инвариантны относительно отображсния $W$, m.e. $W(A) \subseteq A, W(B) \subseteq B, W(C) \subseteq C$.

ДокАЗАТЕЛЬСТво. Обозначим

$$
G_{1}(x, y)=G_{1}\left(x, y, \theta_{1}, \theta_{2}\right)=\frac{y}{x} \frac{\theta_{1}^{2}+2 \theta_{1} \theta_{2}^{2} x+\theta_{2}^{2} x^{2}}{1+2 \theta_{1} \theta_{2}^{2} y+\theta_{1}^{2} \theta_{2}^{2} y^{2}} .
$$

Тогда из (6) получим

$$
\left\{\begin{array}{l}
y_{1}^{+}=G_{1}\left(x_{2}^{-}, x_{2}^{+}\right) F_{1}\left(x_{1}^{-}, x_{1}^{+}\right), \\
y_{1}^{-}=\left[G_{1}\left(\frac{1}{x_{2}^{+}}, \frac{1}{x_{2}^{-}}\right)\right]^{-1} F_{1}\left(x_{1}^{-}, x_{1}^{+}\right), \\
y_{2}^{+}=G_{1}\left(x_{1}^{-}, x_{1}^{+}\right) F_{1}\left(x_{2}^{-}, x_{2}^{+}\right), \\
y_{2}^{-}=\left[G_{1}\left(\frac{1}{x_{1}^{+}}, \frac{1}{x_{1}^{-}}\right)\right]^{-1} F_{1}\left(x_{2}^{-}, x_{2}^{+}\right) .
\end{array}\right.
$$

Заметим, что отображение $F_{1}$ удовлетворяет равенству

$$
F_{1}\left(\frac{1}{y}, \frac{1}{x}\right)=\frac{1}{F_{1}(x, y)}
$$

Обозначим $V\left(x, y, z, t, \theta_{1}, \theta_{2}\right)=G_{1}(x, y) F_{1}(z, t)$. Тогда из $(7)$ получим

$$
\left\{\begin{array}{l}
y_{1}^{+}=V\left(x_{2}^{-}, x_{2}^{+}, x_{1}^{-}, x_{1}^{+}, \theta_{1}, \theta_{2}\right), \\
\frac{1}{y_{1}^{-}}=V\left(\frac{1}{x_{2}^{+}}, \frac{1}{x_{2}^{-}}, \frac{1}{x_{1}^{+}}, \frac{1}{x_{1}^{-}}, \theta_{1}, \theta_{2}\right), \\
y_{2}^{+}=V\left(x_{1}^{-}, x_{1}^{+}, x_{2}^{-}, x_{2}^{+}, \theta_{1}, \theta_{2}\right), \\
\frac{1}{y_{2}^{-}}=V\left(\frac{1}{x_{1}^{+}}, \frac{1}{x_{1}^{-}}, \frac{1}{x_{2}^{+}}, \frac{1}{x_{2}^{-}}, \theta_{1}, \theta_{2}\right) .
\end{array}\right.
$$

Отсюда очевидно, что множества $A, B, C$ инвариантны относительно $W$. Теорема доказана.

Обозначим сужения оператора $W$ на множества $A, B, C$ через $W_{A}, W_{B}, W_{C}$, соответственно.

Пусть

$$
A_{1}=\left\{(x, y) \in \mathbb{R}^{2}: x=\frac{1}{y}\right\} B_{1}=\left\{(x, y) \in \mathbb{R}^{2}: x=y\right\}
$$


ТеОрема 3. Множества $A_{1}, B_{1}, A_{1}$ являются инвариантными относительно $W_{A}, W_{B}, W_{C}$, соответственно, т.е.

$$
W_{A}\left(A_{1}\right) \subseteq A_{1}, \quad W_{B}\left(B_{1}\right) \subseteq B_{1}, \quad W_{C}\left(A_{1}\right) \subseteq A_{1}
$$

ДоКАЗАТЕЛЬСТво. Из (8) Заметим, что

$$
\begin{gathered}
W_{A}:\left\{\begin{array}{l}
y_{1}^{+}=V\left(x_{1}^{-}, x_{1}^{+}, x_{1}^{-}, x_{1}^{+}, \theta_{1}, \theta_{2}\right)=V_{A}\left(x_{1}^{-}, x_{1}^{+}\right), \\
\frac{1}{y_{1}^{-}}=V\left(\frac{1}{x_{1}^{+}}, \frac{1}{x_{1}^{-}}, \frac{1}{x_{1}^{+}}, \frac{1}{x_{1}^{-}}, \theta_{1}, \theta_{2}\right)=V_{A}\left(\frac{1}{x_{1}^{+}}, \frac{1}{x_{1}^{-}}\right),
\end{array}\right. \\
W_{B}:\left\{\begin{array}{l}
y_{1}^{+}=V\left(\frac{1}{x_{2}^{+}}, x_{2}^{+}, \frac{1}{x_{1}^{+}}, x_{1}^{+}, \theta_{1}, \theta_{2}\right)=V_{B}\left(x_{2}^{+}, x_{1}^{+}\right), \\
y_{2}^{+}=V\left(\frac{1}{x_{1}^{+}}, x_{1}^{+}, \frac{1}{x_{2}^{+}}, x_{2}^{+}, \theta_{1}, \theta_{2}\right)=V_{B}\left(x_{1}^{+}, x_{2}^{+}\right),
\end{array}\right. \\
W_{C}:\left\{\begin{array}{l}
y_{1}^{+}=V\left(\frac{1}{x_{1}^{+}}, \frac{1}{x_{1}^{-}}, x_{1}^{-}, x_{1}^{+}, \theta_{1}, \theta_{2}\right)=V_{C}\left(x_{1}^{-}, x_{1}^{+}\right), \\
y_{1}^{-}=V\left(x_{1}^{-}, x_{1}^{+}, \frac{1}{x_{1}^{+}}, \frac{1}{x_{1}^{-}}, \theta_{1}, \theta_{2}\right)=V_{C}\left(\frac{1}{x_{1}^{+}}, \frac{1}{x_{1}^{-}}\right) .
\end{array}\right.
\end{gathered}
$$

Легко видеть, что равенства (9) выполняются. Теорема доказана.

Заметим, что если $\theta_{2}=1\left(J_{2}=0\right)$, то

$$
F_{1}\left(x, y, \theta_{1}, 1\right)=1, \quad G_{1}\left(x, y, \theta_{1}, 1\right)=\frac{y}{x}\left(\frac{\theta_{1}+x}{1+\theta_{1} y}\right)^{2}
$$

и

$$
G_{1}\left(\frac{1}{y}, \frac{1}{x}, \theta_{1}, 1\right)=\frac{1}{G_{1}\left(x, y, \theta_{1}, 1\right)} .
$$

Оператор $W$ при $\theta_{2}=1$ принимает вид

$$
\left\{\begin{array}{l}
y_{1}^{+}=G_{1}\left(x_{2}^{-}, x_{2}^{+}, \theta_{1}, 1\right), \\
y_{1}^{-}=G_{1}\left(x_{2}^{-}, x_{2}^{+}, \theta_{1}, 1\right), \\
y_{2}^{+}=G_{1}\left(x_{1}^{-}, x_{1}^{+}, \theta_{1}, 1\right), \\
y_{2}^{-}=G_{1}\left(x_{1}^{-}, x_{1}^{+}, \theta_{1}, 1\right) .
\end{array}\right.
$$

Из (10) следует

Теорема 4. 1. При $\theta_{2}=1$ оператор $W$, кроме инвариантных мнохсеств $A$, $B, C$, имеет еще инвариантное множество

$$
D=\left\{\left(x_{1}^{-}, x_{1}^{+}, x_{2}^{-}, x_{2}^{+}\right) \in \mathbb{R}^{4}: x_{1}^{-}=x_{1}^{+}, x_{2}^{-}=x_{2}^{+}\right\} .
$$

2. При $\theta_{2}=1$ сужение оператора $W$ на $D$ имеет два инвариантных множества

$$
D_{1}=\left\{(x, y) \in \mathbb{R}^{2}: x=y\right\}, \quad D_{2}=\left\{(x, y) \in \mathbb{R}^{2}: x=\frac{1}{y}\right\}
$$




\section{4. ОБ УРАВНЕНИЯХ НЕПОДВИЖНЫХ ТОЧЕК СУЖЕНИЙ OПЕРАTOPA $W$ HA MHOЖЕCTBA $A_{1}, B_{1}, D_{1}, D_{2}$}

Сужения оператора $W$ на $A_{1}$ и $B_{1}\left(\right.$ при $\theta_{2} \neq 1$ ) приводят к одному и тому же уравнению

$$
x=V\left(x, \frac{1}{x}, x, \frac{1}{x}, \theta_{1}, \theta_{2}\right) .
$$

Его решение дает только трансляционно-инвариантные распределения Гиббса, построенные, в частности, в работе [5].

Сужение оператора $W$ на $D_{1}$ (при $\theta_{2}=1$ ) приводит к уравнению

$$
x=V\left(x, x, x, x, \theta_{1}, 1\right),
$$

решение которого дает трансляционно-инвариантные распределения Гиббса, полностью построенные в работе [2].

Сужение оператора $W$ на $D_{2}$ (при $\left.\theta_{2}=1\right)$ приводит к уравнению

$$
x=V\left(\frac{1}{x}, \frac{1}{x}, x, x, \theta_{1}, 1\right),
$$

решение которого дает нетрансляционно-инвариантные периодические распределения Гиббса, полностью построенные в работе [3].

\section{5. ОБ УРАВНЕНИЯХ НЕПОДВИЖНЫХ ТОЧЕК СУЖЕНИЙ ОПЕРАТОРА $W$ НА МНОЖЕСТВА $A, B, C, D$}

Сужение на $D$ имеет вид

$$
\left\{\begin{array}{l}
x_{1}=\left(\frac{\theta_{1}+x_{2}}{1+\theta_{1} x_{2}}\right)^{2}, \\
x_{2}=\left(\frac{\theta_{1}+x_{1}}{1+\theta_{1} x_{1}}\right)^{2} .
\end{array}\right.
$$

Система (14) дает периодические распределения Гиббса для обычной $\left(J_{2}=0\right)$ модели Изинга (см. [2]).

Сужение на $B$ имеет вид

$$
\left\{\begin{array}{l}
x=\frac{\theta_{1}^{2} y^{2}+2 \theta_{1} \theta_{2}^{2} y+\theta_{2}^{2}}{\theta_{1}^{2} \theta_{2}^{2} y^{2}+2 \theta_{1} \theta_{2}^{2} y+1}, \\
y=\frac{\theta_{1}^{2} x^{2}+2 \theta_{1} \theta_{2}^{2} x+\theta_{2}^{2}}{\theta_{1}^{2} \theta_{2}^{2} x^{2}+2 \theta_{1} \theta_{2}^{2} x+1} .
\end{array}\right.
$$

Обозначим $\theta=\theta_{2}^{2}, u=\theta_{1} x, v=\theta_{1} y$. Тогда из (15) имеем

$$
\left\{\begin{array}{l}
\theta u v^{2}+2 \theta u v+u-\theta_{1} v^{2}-2 \theta \theta_{1} v-\theta \theta_{1}=0, \\
\theta v u^{2}+2 \theta u v+v-\theta_{1} u^{2}-2 \theta \theta_{1} u-\theta \theta_{1}=0 .
\end{array}\right.
$$

Обозначим $\sigma_{1}=u+v, \sigma_{2}=u v$. Система (16) распадается на две системы

$$
\begin{aligned}
& \left\{\begin{array}{l}
\theta \sigma_{1} \sigma_{2}+4 \theta \sigma_{2}+\sigma_{1}-\theta_{1}\left(\sigma_{1}^{2}-2 \sigma_{2}\right)-2 \theta \theta_{1} \sigma_{1}-2 \theta \theta_{1}=0, \\
4 \sigma_{2}=\sigma_{1}^{2},
\end{array}\right. \\
& \left\{\begin{array}{l}
\theta \sigma_{1} \sigma_{2}+4 \theta \sigma_{2}+\sigma_{1}-\theta_{1}\left(\sigma_{1}^{2}-2 \sigma_{2}\right)-2 \theta \theta_{1} \sigma_{1}-2 \theta \theta_{1}=0, \\
\theta \sigma_{2}=\theta_{1} \sigma_{1}+2 \theta \theta_{1}+1 .
\end{array}\right.
\end{aligned}
$$


Подставляя $\sigma_{2}=\sigma_{1}^{2} / 4$ в первое уравнение системы (17), получим

$$
\theta \sigma_{1}^{3}+2 \theta \sigma_{1}^{2}+4\left(1-2 \theta \theta_{1}\right) \sigma_{1}-8 \theta \theta_{1}=0 .
$$

Заметим, что это уравнение можно полностью решить, но оно дает только трансляционно-инвариантные распределения Гиббса.

Подставляя $\sigma_{2}=\theta^{-1}\left(\theta_{1} \sigma_{1}+2 \theta \theta_{1}+1\right)$ в первое из уравнений $(18)$, получим

$$
\sigma_{1}=-\frac{6 \theta^{2} \theta_{1}+4 \theta \theta_{1}^{2}+4 \theta+2 \theta_{1}}{2 \theta_{1}^{2}+4 \theta \theta_{1}+2 \theta}<0 .
$$

Но по построению $\sigma_{1}=u+v>0$. Таким образом, система (18) не имеет положительных решений.

Сужение оператора $W$ на множество $A$ можно привести к виду

где

$$
\left\{\begin{array}{l}
x=\frac{\varphi(x) \psi(x)}{\varphi(y)}=K(x, y) \\
y=\frac{\varphi(y) \psi(y)}{\varphi(x)}=K(y, x)
\end{array}\right.
$$

$$
\varphi(x)=\frac{x}{1+2 \theta_{1} \theta_{2}^{2} x+\theta_{1}^{2} \theta_{2}^{2} x^{2}}, \quad \psi(x)=\frac{\theta_{2}^{2}+2 \theta_{1} \theta_{2}^{2} x+\theta_{1}^{2} y^{2}}{1+2 \theta_{1} \theta_{2}^{2} x+\theta_{1}^{2} \theta_{2}^{2} x^{2}} .
$$

Система (20) эквивалентна системе

$$
\left\{\begin{array}{l}
x+y=K(x, y)+K(y, x) \\
x-y=K(x, y)-K(y, x) .
\end{array}\right.
$$

Легко проверить, что система (21) распадается на две:

$$
\begin{aligned}
& \left\{\begin{array}{l}
x+y=K(x, y)+K(y, x), \\
x=y,
\end{array}\right. \\
& \left\{\begin{array}{l}
x+y=K(x, y)+K(y, x), \\
S(x, y)=0,
\end{array}\right.
\end{aligned}
$$

где $S(x, y)$ - некоторая функция такая, что $S(x, y)=S(y, x)$ для любых $x, y$.

Заметим, что система (22) дает трансляционно-ивариантные распределения Гиббса. Система (23) симметрична относительно перестановки $x, y$, поэтому можно ввести замену $\sigma_{1}=x+y, \sigma_{2}=x y$. Тогда система (23) примет более простой вид, и второе уравнение будет иметь степень 3 относительно $\sigma_{1}$, т.е. можно будет получить полное решение. Но, подставляя его в первое из уравнений (23), получаем громоздкое уравнение высокого $(>3)$ порядка, решить которое аналитическими методами не удается.

Сужение на $C$ имеет вид

$$
\left\{\begin{array}{l}
x=\frac{a(y) b(x)}{\psi\left(x^{-1}\right)} \\
y=a(y) b(x) \psi(y)
\end{array}\right.
$$

где

$$
a(x)=x\left(\theta_{1}^{2} x^{2}+2 \theta_{1} \theta_{2}^{2} x+\theta_{2}^{2}\right), \quad b(x)=\left(x\left(x^{2}+2 \theta_{1} \theta_{2}^{2} x+\theta_{1}^{2} \theta_{2}^{2}\right)\right)^{-1} .
$$

Первое уравнение ситемы (24) есть кубическое уравнение относительно $y$, второе - относительно $x$. Их можно решить. Подставляя полученное решение первого уравнения во второе и наоборот, получаем достаточно громоздкие уравнения высокого (> 3) порядка, решить которые аналитическими методами не удается. 


\section{6. ОБСУЖДЕНИЕ. ОТКРЫТЫЕ ПРОБЛЕМЫ}

Одна из основных задач для данного гамильтониана - описание множества всех соответствуюших ему гиббсовских распределений. Но полностью описать это множество пока не удается даже для достаточно простых гамильтонианов. В работе [6] модель (1) исследована на физическом уровне; там при конкретных значениях параметров $J_{1}, J_{2}$ дана фазовая диаграмма состояний системы.

Но интересно получить область параметров $J_{1}, J_{2}$, где существуют неединственные распределения Гиббса. В работе [5] решена задача описания трансляционно-инвариантных гиббсовских мер модели (1). Результаты настояшей работы позволяют явно выписать области значения параметров $J_{1}, J_{2}$, где существуют периодические распределения Гиббса. В сужении оператора $W$ на множество $B$ доминирующую роль играет параметр $J_{2}$, т.е. если $J_{2}=0$, то система (15) имеет единственное решение $x=y=1$. Но если $J_{2} \neq 0$, то сушествует область параметров $J_{1}, J_{2}$, при которых система (15) имеет более чем одно решение. Эта область параметров получается при решении кубического уравнения (19).

Сужениям оператора $W$ на инвариантные множества $A$ и $C$ соответствуют очень громоздкие уравнения. Их решения можно получить с помощью компьютера, но это не входит в круг наших интересов.

Заметим, что связь между решениями уравнений и распределениями Гиббса задается равенствами (2) и (4). Сужения оператора на множества $A_{1}, B_{1}, D, D_{1}, D_{2}$ дают известные распределения Гиббса. Сужения оператора на множества $A, B, \mathrm{C}$ приводят к новым распределениям Гиббса.

И, наконец, сформулируем проблемы, которые оказались достаточно сложными и требующими дальнейшего рассмотрения.

1. Сушествуют ли другие инвариантные множества оператора $W$ ?

2. Сушествуют ли неподвижные точки вне инвариантных множеств?

3 . Существует ли подгруппа $G_{k}^{*}$ конечного индекса группы $G_{k}$, относительно которой функциональное уравнение $(11)$ на классе $G_{k}^{*}$-периодических функций решается полностью?

\section{Список литературы}

[1] Н. Н. Ганиходжаев. ДАН РУз. 1994. № 4. С. 3-5.

[2] Н. Н. Ганиходжаев, У. А. Розиков. ТМФ. 1997. Т. 111. № 1. С. 109-117.

[3] У. А. Розиков. ТМФ. 1997. Т. 112. № 1. С. 170-176.

[4] У. А. Розиков. ТМФ. 1999. Т. 118. № 1. С. 95-104.

[5] Н. Н. Ганиходжаев, У. А. Розиков. УзМЖ. 1995. № 2. С. 36-47.

[6] S. Katsura, M. Takizawa. Progr. Theor. Phys. 1974. V. 51. № 1. P. 82-98.

Поступила в редакцию 22.V.2002 г. 